\title{
Late Complications of Clinical Clostridium Histolyticum Collagenase Use in Dupuytren's Disease
}

\author{
Warren M. Rozen ${ }^{1,2 *}$, Yasith Edirisinghe ${ }^{1,2}$, John Crock $^{1,2}$
}

1 Department of Plastic and Reconstructive Surgery, Dandenong Hospital, Southern Health, Dandenong, Victoria, Australia, 2 Department of Surgery, Faculty of Medicine, Monash University, Clayton, Victoria, Australia

\begin{abstract}
Introduction: While Dupuytren's disease can cause disabling contractures requiring open surgery, a less-invasive option using Clostridium Histolyticum collagenase $(\mathrm{CHC})$ via percutaneous injection was recently reported. A recent prospective, randomized trial demonstrated few complications during 90 days follow-up, however did not assess any longer term followup for these patients. Long-term outcomes in this setting have not been adequately reported, and the current manuscript aims to identify late complications from the clinical use of percutaneous CHC.

Methods: The current manuscript reports an extended 12-month follow-up for a cohort of twelve of patients enrolled in the original prospective, randomized trial, treated at a single institution. An analysis of complications requiring surgical intervention was undertaken.

Results: Two of twelve patients reported debilitating pain and triggering requiring surgical intervention. Extensive deeptissue scarring and adhesions were identified, providing the first visual and qualitative analysis of the pathologic effects of $\mathrm{CHC}$.

Conclusion: Late complications from CHC use can and have occurred, outside the follow-up period of the initial phase III trials. Longer term follow-up of such patients is thus essential, and further investigation and characterization of the late effects of $\mathrm{CHC}$ use is warranted.
\end{abstract}

Citation: Rozen WM, Edirisinghe Y, Crock J (2012) Late Complications of Clinical Clostridium Histolyticum Collagenase Use in Dupuytren's Disease. PLoS ONE 7(8): e43406. doi:10.1371/journal.pone.0043406

Editor: Hani A. Awad, University of Rochester, United States of America

Received May 8, 2012; Accepted July 23, 2012; Published August 17, 2012

Copyright: (c) 2012 Rozen et al. This is an open-access article distributed under the terms of the Creative Commons Attribution License, which permits unrestricted use, distribution, and reproduction in any medium, provided the original author and source are credited.

Funding: The authors have no support or funding to report.

Competing Interests: The authors have declared that no competing interests exist.

* E-mail: warrenrozen@hotmail.com

\section{Introduction}

Dupuyten's disease is a fibroblastic proliferative disorder in which normal fascial bands of the palmar fascia become diseased cords, which shorten progressively leading to soft-tissue and joint contractures. With limited conservative measures available, the only definitive management option for disabling Dupuyten's disease has been surgical intervention, ranging from percutaneous cordotomy to radical dermo-fasciectomy.

A new drug containing Clostridium Histolyticum collagenase (CHC) has been reported to be capable of lysing Dupuytren's cords and is being marketed as a product that can be used to treat Dupuyten's disease in an office-based setting by simply injecting the product subcutaneously into the affected cords (Xiaflex, Auxillium Pharmaceuticals) [1-4]. This product has entered clinical use, largely based on phase III trials with the 'Collagenase Option for Reduction of Dupuytren's (CORD1 and CORD2)' studies reporting high efficacy and low complication rates. One cohort of these patients were tested in a single centre in Melbourne Australia, and in this group, longer follow-up outside of the study period was able to be assessed.

While a low complication rate was reported within initial trials, in which a 90 day follow-up period was included in the study protocols, we were able to achieve longer term follow-up
(12 months) for 12 patients, and were able to identify some significant complications which were not previously recognised.

\section{Methods}

\section{Ethics Statement}

Inclusion of patients in this study was approved by the institutional ethical committee of Southern Health and the National Health and Medical Research Council of Australia guidelines. The study complies with the Declaration of Helsinki, 1995. The subjects gave full written informed consent, and patient anonymity has been preserved. This institutional review board specifically approved this study.

A cross-sectional study was undertaken of a selected group of patients from the cohort of participants included in the phase III trials of CHC use as described in the CORD1 and CORD2 studies, comprising prospective, randomized, double-blind, placebo-controlled, multicentre trials [1]. The cohort of patients comprised 12 patients who had all been treated at a single institution. CORD1 and CORD2 trial protocols for recruitment, consent, randomization, treatment and initial follow-up had been adhered to, and as such, the patients included were patients with Dupuyten's disease who had fixed-flexion contractures of the 
metacarpophalangeal joint or proximal interphalangeal joint of 20 degrees or more in one finger, were of good health, and were able to appropriately consent. Exclusion criteria included bleeding disorders or anticoagulant use, and previous treatment of the primary joint. Written informed consent was provided in all cases. The primary intervention for each of the 12 patients had been the administration of up to three CHC injections at 1-month intervals, together with early mobilisation. CHC dosing comprised $0.58 \mathrm{mg}$ per injection reconstituted in $0.39 \mathrm{ml}$ of sterile saline for metacarpophalangeal joints or $0.31 \mathrm{ml}$ of sterile diluent (a combination of sterile saline and calcium chloride) for proximal interphalangeal joints. After reconstitution, $0.25 \mathrm{ml}$ was injected into a cord affecting an metacarpophalangeal joint and $0.20 \mathrm{ml}$ injected into a cord affecting a proximal interphalangeal joint. The injections were applied subcutaneously, directly into the affected cords.

While initial reporting of the CORD studies reported 90 day outcomes, the current study reports the 12 month follow-up of the cohort described. All early-term follow-up results were as reported in the original trial, with the reported adverse effects in this cohort being pain/discomfort during the action of the CHC in dividing the Dupuytren's cords, insignificant bruising and pain around the injection sites. Furthermore, all twelve patients achieved a full range of flexion and extension, as per the end criteria of the CORD1 and CORD2 trials. However, the primary endpoint of this study was the incidence of complications requiring surgical intervention, presenting outside the initial follow-up period. The findings of surgical exploration following CHC use has not been described, and this study provides a visual and qualitative analysis of the pathologic effects of CHC use.

\section{Results}

Within the extended follow-up period of the current study, between 3 and 12 months post-injection, 10 of the 12 patients did not report any further complications. Two patients however presented with significant disability from painful triggering of the injected finger. Both patients were male, with no significant past or present comorbidities or confounding factors. For both patients, their symptoms were managed conservatively throughout the study period, with monthly medical assessment, and conservative therapy comprising mobilisation, night splinting and scar management. At each of the monthly follow-up measurements, these 2 patients recorded full range of motion in flexion and extension, despite the clinically evident persistence of pain and stiff fingers. By 12 months, it was evident that these patients had no further functional recovery and with a clinical diagnosis of significant scarring/adhesions, operative intervention was undertaken. Similar clinical findings were experienced with both patients.

Each patient underwent surgical exploration for both diagnosis and management, with the aim of confirming the pathology, determining the anatomical structures involved and managing the pathology through fasciectomy, tenolysis and soft tissue reconstruction as required. This intervention also served as a means to assess the anatomy and changes caused by CHC injection, an assessment not previously reported, and to photographically record these effects.

Figures 1, 2, 3 demonstrate the photographic sequence of dissection from superficial to deep, with findings consistent between both cases. Superficial dissection demonstrated the presence of an ongoing pretendinous band of Dupuyten's disease, albeit attenuated, confirming the incomplete action of CHC on the disease (Figure 1). Deeper dissection, to a plane immediately deep to the superficial palmar fascia, revealed dense adhesions around the flexor tendon and a thickened Al pulley distally. The adhesions extended laterally around the flexor tendons to involve the digital neurovascular bundles circumferentially, outside a plane involved by Dupuyten's disease (Figure 2). Deeper dissection still identified further dense adhesions and flexor tendon scarring, limiting tendon glide. This scar tissue was within the tendon sheath, surrounding the tendons themselves, however both flexor digitorum superficialis and flexor digitorum profundus tendons were intact. Both patients underwent neurolysis, tenolysis, adhesiolysis, fasciectomy and closure. Histopathologic assessment of the excised tissue confirmed dense scar tissue. Longer term follow-up, 18 months after CHC injection and 6 months after revision surgery, demonstrated a return to full range of motion and no need for subsequent intervention.

\section{Discussion}

Dupuytren's disease has been an extensively investigated disease process throughout the $19^{\text {th }}$ and $20^{\text {th }}$ centuries, with even the earliest reference to Dupuyten's disease in 1614 by Felix Plater highlighting the debility caused by a condition causing flexion in the little fingers, which he postulated to be caused by flexor tendon shortening [5]. In 1777, Sir Henry Cline demonstrated the causal relationship of palmar fascia to the condition from a series of cadaveric dissections, followed by Baron Guillaume Dupuytren in 1831 comprehensively describing the differential diagnosis, suspected pathology, clinical course, and management of the disease. Since that time, the etiology, pathophysiology and differing management strategies have been extensively studied by various research groups [6]. These have highlighted the more common associations with the disease: men are more likely to have Dupuyten's disease than women, with 9 of every 10 patients being male; the incidence increases with age; the disease is common in Scandinavia, Great Britain, Ireland, Australia, and North America, while it is uncommon in southern Europe and South America, and rare in Africa and China. Other studies have shown associations with diabetes, alcoholism and hypercholesterolemia, and twin and genetic studies conducted thus far show a strong genetic influence, with current evidence supporting an autosomal dominant inheritance pattern with variable penetrance $[7,8]$.

With limited conservative measures available, the mainstay of management options for disabling Dupuyten's disease has been

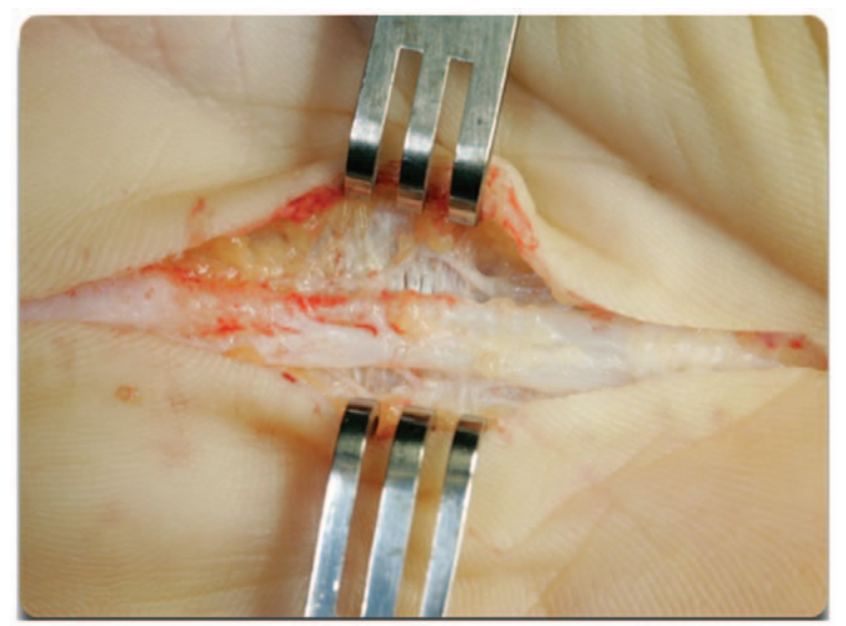

Figure 1. Superficial dissection revealing residual attenuated pretendinous Dupuytren's cord.

doi:10.1371/journal.pone.0043406.g001 


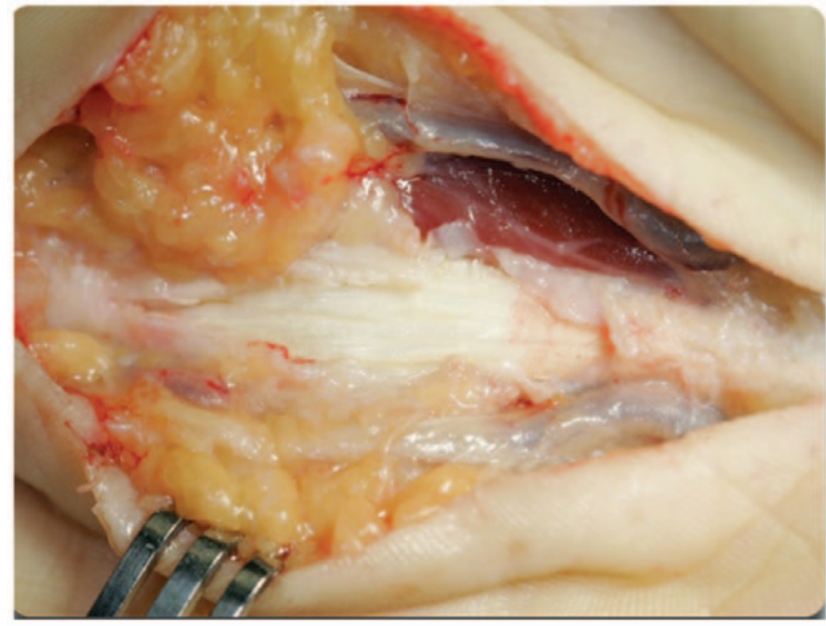

Figure 2. Deeper dissection through dense peritendinous adhesions and scarring.

doi:10.1371/journal.pone.0043406.g002

surgery, with McGrouther et al in 2006 publishing a comprehensive algorithm for the surgical management of Dupuyten's disease [9]. This strategy involves clear identification of symptoms on presentation and close follow-up for a year, with guidelines recommending operative intervention if clear progression of the condition or clear deterioration of function are observed. Operative intervention can vary depending on the degree of involvement of the disease, and may involve percutaneous aponeurotomy, limited fasciectomy or dermofasciectomy for more extensive disease.

The introduction of CHC boasted a revolutionary therapy in the treatment of Dupuyten's disease, suggesting a minimally invasive means to releasing palmar fascial contractures with good efficacy and a safe side-effect profile [1-4]. The compound, isolated from the culture medium of Clostridium Histolyticum, comprises a fixed-ratio mixture of a clostridial type-I collagenase (termed by the manufacturer as AUX-I) and a clostridial type-II collagenase (AUX-II). The type-I collagenase is a $114-\mathrm{kDa}$ single polypeptide chain containing approximately 1000 amino acids of known sequence. The type-II collagenase is $113 \mathrm{kDa}$ in weight and is also approximately 1000 amino acids long. These enzymes differ from each other in terms of domain structure, substrate affinity, catalytic efficiency, and preferred cleavage site on the collagen molecule. According to the pre-clinical studies conducted by the manufacturer, these class-I and class-II collagenases work synergistically rather than independently [3].

The form and pharmacokinetics of the compound is straightforward, with in-vivo and in-vitro studies suggesting that the compound can lyse Dupuytren's cords while leaving the adjacent neurovascular structures unaffected [10]. CHCs are metalloprotease enzymes of the matrixin subfamily, which function via lysis of the three-dimensional structure of collagen molecules. The CHC molecule has demonstrated in-vivo and in-vitro catalytic activity against all collagen types, except for collagen type IV. Dupuytren's cords show an abundance of type 1 collagen as well as unusually large amounts of collagen type III, which is otherwise not commonly seen within palmar fascia. The basement membrane of the neurovascular structures mostly contains type IV collagen, and is therefore not affected even in the case of direct injection [10]. Non-clinical studies failed to show significant degradation of blood vessels, nerves and epithelia following local injection, which may be related to its poor activity against type IV collagen [3].

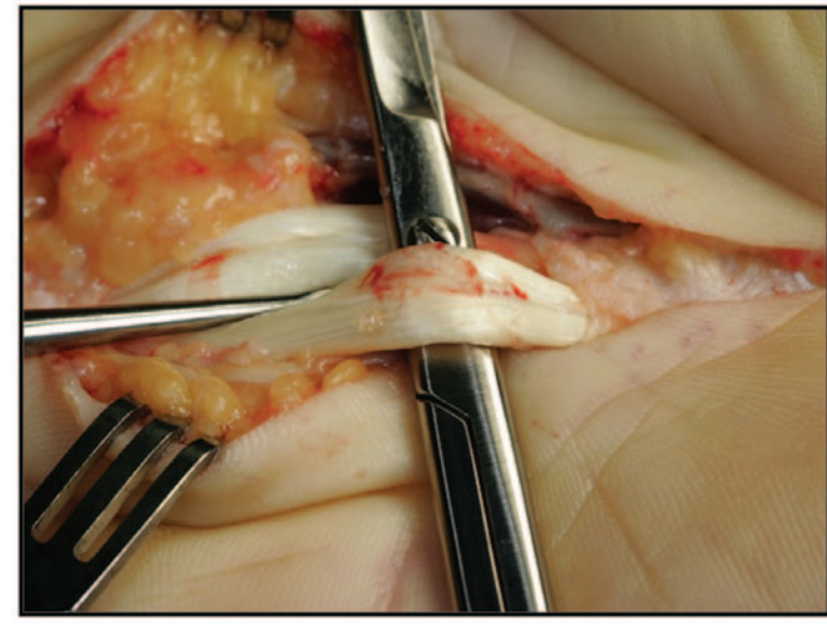

Figure 3. Further dissection revealing dense scarring of the flexor tendons with adhesions limiting tendon glide. doi:10.1371/journal.pone.0043406.g003

Pre-clinical animal studies did not reveal any detectable systemic effects following intravenous injection of collagenase, and this has been supported by clinical trials that have not shown any detectable levels of collagenase systemically after its subcutaneous injection [11]. Consequently, clinical drug interaction studies have not been conducted. Of note, the tetracycline family of antibiotics has been shown to inhibit matrix metalloproteinasemediated collagen degradation in published in-vitro experiments, which suggests that concomitant use of tetracyclines may inhibit collagenase action [12]. Other complications have included reports of local inflammatory reactions at the injection site as a result of vascular leakage and neutrophil chemotaxis [10,11].

The CORD1 trial reported the clear success of $\mathrm{CHC}$ injections for treating Dupuyten's disease. In this trial, 3 successive injections spaced 30 days apart, followed by aggressive early mobilization and night splinting, was able to achieve post-injection extension to 5 degrees or less in $64 \%$ of the cases, while the placebo arm achieved this extension in $6.8 \%$ of the cases. This benefit was associated with a relatively low side-effect profile, with reports of local immune/inflammatory reactions such as pruritis, injection site pain, lymphadenopathy, ecchymosis, erythema, and blistering. Two cases of flexor tendon rupture were reported, with the cause for these 2 cases identified as suboptimal injection technique. With a corrected technique, the extension of the study reported 3 flexor tendon ruptures out of 2600 injections [1].

The combined analysis of outcomes and complications from the CORD1 and CORD2 studies suggested that anatomically, the CHC technique employed resulted in effects on the diseased palmar fascia and some action on the flexor tendon. The current study highlighted several key outcomes. Firstly, tendon scarring and adhesions causing painful triggering of the affected flexor tendon is a noteworthy late complication that is worthy of assessment and consideration in the consent process, and may require revisionary surgery. Secondly, it is clear that the action of CHC is more anatomically extensive than previously considered. Post-injection adhesions were evident lateral to the flexor tendons, within the flexor sheath (at the Al pulley) and circumferentially around the neurovascular structures of the digits. In addition to the presenting complaints described, the longer term impact on digital function or on surgical outcomes in recurrent disease, is yet to be established. Combining the flexor tendon ruptures reported in the CORD1 study and the extensive adhesions presented in this 
study, alteration of CHC dosing or drug concentration, injection technique or image-guidance during the injection, are all techniques worth investigating as a means to improved outcomes.

\section{Conclusions}

While the use of CHC can achieve excellent results in most patients, complications can occur outside of early follow-up, such as occurred in initial phase III trials. Late complications include flexion pain and triggering, and may require operative intervention as late as 12 months post-injection. With operative findings of deep tissue scarring and tendon adhesions following

\section{References}

1. Hurst LC, Badalamente MA, Hentz VR, Hotchkiss RN, Kaplan FT, et al (2009) Injectable collagenase clostridium histolyticum for Dupuytren's contracture. N Eng J Med 361 (10): 968-979.

2. Amadio PC (2009) What's new in hand surgery. J Bone Joint Surg (Am) 91: 496502.

3. Desai SS, Hentz VR (2010) Collagenase clostridium histolyticum for Dupuytren's contracture. Expert Opin Biol Therapy 10(9): 1395-1404.

4. Badalamente MA, Hurst LC (2007) Efficacy and safety of injectable mixed collagenase subtypes in the treatment of Dupuytren's contracture. J Hand Surg (Am) 32: 767-774.

5. Saar JD, Grothaus PC (2000) Dupuytren's Disease: An Overview. Plas Reconstr Surg 106(1): 125-134.

6. Rayan GM (2007) Dupuytren's Disease: Anatomy, Pathology, Presentation and Treatment. J Bone Joint Surg (Am) 89 (1): 190-198.
CHC use, the likely mechanism for these complications is through a broad anatomical action of $\mathrm{CHC}$ on palmar and digital anatomy, and these effects warrant further investigation and characterization.

\section{Author Contributions}

Conceived and designed the experiments: WMR YE JC. Performed the experiments: WMR YE JC. Analyzed the data: WMR YE JC. Contributed reagents/materials/analysis tools: WMR YE JC. Wrote the paper: WMR YE JC. Surgical planning and execution: WMR YE JC.

7. Townley WA, Baker R, Grobbelaar AO (2006) Dupuytren's contracture unfolded. BMJ 332: 397-400.

8. Hindocha S, McGrouther DA (2009) Epidemiological Evaluation of Dupuytren's Disease - Incidence and Prevalence Rates in Relation to Etiology. Hands 4: 256-269.

9. Bayat A, McGrouther DA (2006) Management of Dupuytren's disease - Clear advise for an elusive condition. Ann Roy Coll Surg Engl 88: 3-8.

10. Rydevik B, Ehira T, Linder L, Olmarker K, Romanus M, et al (1989) Microvascular response to locally injected collagenase. Scand J Plast Reconstr Surg 23: 17-21.

11. Badalamente MA, Hurst LC, Hentz VR (2002) Collagen as a clinical target: nonoperative treatment of Dupuytren's disease. J Hand Surg (Am) 27: 788-798.

12. Suomalainen K, Sorsa T, Golub L, Ramamurthy N, Lee HM, et al (1992) Specificity of the anticollagenase action of tetracyclines; relevance to their antiinflammatory potential. Antimicr Agents Chemotherapy 36: 227-229. 